\title{
Performance Analysis of Ensemble of Long Irregular LDPC Code over various Channels with Cut off Rate
}

\author{
Madhusmita Mishra \\ Dept. of Electronics and \\ Communication \\ National Institute Of \\ Technology \\ Rourkella-769008, India
}

\author{
Sarat Kumar Patra \\ Dept. of Electronics and \\ Communication \\ National Institute Of \\ Technology \\ Rourkella-769008, India
}

\author{
Ashok Kumar Turuk \\ Dept. of Computer Science \\ Engineering \\ National Institute Of \\ Technology \\ Rourkella-769008, India
}

\begin{abstract}
A long Irregular LDPC code that performs at rates extremely close to the Shannon capacity has been taken. The code has carefully chosen degree patterns. Simulations has been done with Hard and Soft-decision decoding to compare the performance of this code with the rates $1 / 2,1 / 3,1 / 4,2 / 3,2 / 5$, $3 / 4,3 / 5,4 / 5,5 / 6,8 / 9$ and $9 / 10$ over various channels like AWGN channel, Rayleigh fading channel and Rician fading channel. The little dependence of BER performance on various channels is explored here along with the conjecture of the concept of computational cutoff rate that represents an upper limit on rate of transmission for practically instrumentable reliable communications.
\end{abstract}

\section{General Terms}

Orthogonal frequency division multiplexing (OFDM), Quadrature Amplitude Modulation (QAM)

\section{Keywords}

LDPC (Low Density parity check), Upper Bound, Cut off Rate, Bhattacharyya bound, Hard-decision, Soft-decision

\section{INTRODUCTION}

For any channel, the bandwidth, data rate, noise and error rate are related to each other. The greater the bandwidth, the greater will be the cost. All transmission channels of any practical interest are limited in bandwidth due to the constrained physical properties of the transmission medium. For digital data transmission, in order to use bandwidth efficiently it is required to get higher possible data rate at a particular limit of error rate for a given bandwidth and to which the main constraint is the noise. If binary signals are transmitted then the supported data rate will be twice the bandwidth. But, using multilevel signaling the data rate can be increased by a factor of $\log _{2} M$, where $M$ is the no of signal levels [5]. Now as data rate increases, the bits become shorter in duration and as a result more bits are affected by a given pattern of noise concluding the statement that higher data rate leads to higher error rate. The solution now is to increase the signal to noise ratio (SNR), which sets the upper bound on the achievable data rate. Shanon's formula assumes only white noise (thermal noise) and it does not account for the impulse noise or distortion due to attenuation and delay. While the Shanon's formula represents the theoretical maximum that can be achieved, but in practice much lower rates are achieved. Similarly it does not suggest rather provide a yardstick for finding a suitable signal code to achieve error free transmission. Here we have used a long Irregular LDPC code which answers all the questions aroused from Shanon's theorem and it has excellently used the distance properties of LDPC codes[1,2]. Though we can communicate in principle at rate near channel capacity with arbitrarily small error probability, the parameter $R_{c}$ (cut off rate ) represents an upper limit on rate for reliable practical communication $[4,9,10]$. The $R_{c}$ act as a compact figure of merit for a modulation and demodulation system employing channel coding technique. The rest part of the paper is as follows. The second part explores the cut off rate as a means of assessing modulation and coding options. The third part gives the details of encoding and decoding methods used here and discusses simulation results followed by the conclusion section.

\section{CUT OFF RATE TOWARDS ASSESSING MODULATION AND CODING OPTIONS}

In a generic model for the point to point digital communication system, the Information source is modeled probabilistically and messages are viewed as outputs from some random experiments [4]. For the action of the channel on the input signal, a well defined mathematical model is assumed and this includes stochastic and deterministic aspects. In analog systems mean-square error between source and destination waveforms is taken as criteria, whereas the performance is measured by symbol error probability or message error probability in discrete communication. These performance measurement criterions are referred as fidelity criterions. For every combination of source model and fidelity criterion, a rate distortion function can be assigned as $\mathrm{R}(\mathrm{n})$, which is specified in bits per unit of time that depends only on the source description and on the fidelity criterion. The argument $\mathrm{n}$ of the rate distortion function is the smallest expected or average distortion achievable by any system representing the source with $\mathrm{R}(\mathrm{i})$ bits per unit source time. The solution for ' $i$ ' is obtained from

$$
R\left(i^{*}\right)=S
$$

Any system how much complicated it may be, can have an average distortion of less than $i^{*}$. If the $i^{*}$, resulting from above is unacceptably large then we have to render for either providing greater channel capacity $(\mathbf{S})$ or slowing the source symbol production rate. The reason for adopting cut off coding rate is broadly in the sense to achieve highly reliable 
communication at rates approaching channel capacity limit defined by the physical channel .Channel coding is useful in any kind of noisy channel transmission problem and it offers particularly impressive gains on fading channels. If a block code $\mathbf{C}$ has a list of $\mathrm{Q}$ codewords, each an n-tuple, whose entries are from an alphabet of size $\mathrm{k}$, and then assuming that the message source selects messages equiprobably and independently, the entropy of the codeword selection process will be $\log _{2} Q$ bits per message. Hence the exchanged information rate will be given by

$R=\log _{2} Q / n$ bits per codeword symbol

So the no of possible codewords for a given rate will be given by

$$
Q=2^{n R}
$$

Next step here is to reach at a decision of designing an intelligent coded communication system.

\subsection{Concept of Upper Bound}

An upper bound must be set on the evaluation of error probability and the Bhattacharyya bound is such an bound. It is given by below expression:

$$
\begin{aligned}
& P_{2}\left(y_{1} \rightarrow y_{2}\right) \leq \sum_{z} P\left(z / y_{1}\right)^{1 / 2} P\left(z / y_{2}\right)^{1 / 2} \\
& =P_{B}\left(y_{1}, y_{2}\right)
\end{aligned}
$$

Where, $P_{2}\left(y_{1} \rightarrow y_{2}\right)$ is the probability of the event that $y_{1}$ is transmitted, but $y_{2}$ has higher likelihood .The summation here can be interpreted as an n-dimensional sum including all $z^{\prime} s$ and not just those in the decision region meant for the codeword $y_{2} . P_{B}\left(y_{1}, y_{2}\right)$ is the Bhattacharyya bound here and it does not require channel symmetry or memory less behavior. The negative logarithm of Bhattacharyya bound is known as Bhattacharyya distance and is given by

$d_{B}\left(y_{1}, y_{2}\right)=-\log \left[P_{B}\left(y_{1}, y_{2}\right)\right]$

Hence the two codeword upper bound on error probability is

$$
P_{B}\left(y_{1}, y_{2}\right)=2^{-d_{B}\left(y_{1}, y_{2}\right)}
$$

This bound is surprisingly tight for most channel of interest. Now, if the channel is memory less, then denoting the output variable as $\mathrm{Z}$, we can have

$$
P(z / y)=\prod_{j=0}^{n-1} P\left(z_{j} / y_{j}\right)
$$

Now substituting (7) to (4) and expanding the n-fold sum, we can write the bound as a product of scalar summations as below:

$$
P_{B}\left(y_{1}, y_{2}\right)=\prod_{j=0}^{n-1} \sum_{k=0}^{Q-1}\left[P\left(z_{k j} / y_{1 j}\right) P\left(z_{k j} / y_{2 j}\right)\right]^{1 / 2}
$$

Where $Q$ is the no. of possible codewords for a given rate. In this equation the bound for the error probability is a symmetric function of its two arguments. Replacing the quantity inside the summation as $b_{j}$ and mentioning $b_{j}$ as the channel transition probabilities for the jth symbol position, we can notice that $b_{j}$ is a function of the choice of two code symbols. So the bound in more simple form is:

$$
P_{B}\left(y_{1}, y_{2}\right)=\prod_{j=0}^{n-1} b_{j}
$$

\subsection{Concept of Cut off rate $\mathbf{R}_{c}$}

Now, without concentrating on any two specific codewords $\mathrm{y}_{1}$ and $y_{2}$ and doing random selection of two codeword codes from the ensemble of all two codeword codes of length $n$ and assuming code symbols of a given codeword are generated independently, then the probability assigned to n-tuples will be:

$$
P\left(y_{i}\right)=\prod_{j=0}^{n-1} P\left(y_{i j}\right)
$$

Hence, the probability measure assigned to selection of a given code will be:

$$
P\left(y_{1}, y_{2}\right)=P\left(y_{1}\right) P\left(y_{2}\right)=\prod_{j=1}^{n} P\left(y_{1 j}\right) P\left(y_{2 j}\right)(11)
$$

Taking the two codeword to be symmetric in its arguments and replacing $P_{2}\left(y_{1} \rightarrow y_{2}\right)$ by $P\left(y_{1}, y_{2}\right)$, the upper bound on the two codeword error probability with a randomly selected pair of codewords will be the ensemble average error probability as below: 
$\overline{P_{2}\left(y_{1}, y_{2}\right)} \leq$

$\prod_{j=0}^{n-1} \sum_{z_{j}} \sum_{y_{1 j}} \sum_{y_{2 j}} P\left(y_{1 j}\right) P\left(y_{2 j}\right)\left[P\left(z_{j} / y_{1 j}\right) P\left(z_{j} / y_{2 j}\right)\right]^{1 / 2}$

Simplifying the above equation with the fact that each term in the product is independent of position index $\mathrm{j}$ and taking the subscripted variables as dummy variables, we will get the simplified form of above equation as below:

$\overline{P_{2}\left(y_{1}, y_{2}\right)} \leq \prod_{j=0}^{n-1} \sum_{z}\left[\sum_{y} P(y) P(z / y)^{1 / 2}\right]^{2}$

To represent the equation (13) more simple we will define a quantity as:

$R_{c}(P)=-\log _{2}\left(\sum_{z}\left[\sum_{y} P(y) P(z / y)^{1 / 2}\right]^{2}\right)$

Using $R_{c}(P)$, the bound on error probability for the ensemble of two codeword codes is given by

$$
\overline{P_{2}\left(y_{i}, y_{j}\right)} \leq 2^{-n R_{c}(P)}
$$

In a larger code, where $\mathrm{n}$ is large, provided if the codeword probability structure is unchanged, we can generalize the results for any pair of codewords. The distribution on code symbols that means $\mathrm{P}(\mathrm{y})$ can be chosen freely so as to obtain the smallest upper bound. Thus we define $\mathrm{R}_{\mathrm{c}}$ to be

$$
R_{c}=\max _{P(y)}\left\{-\log _{2}\left(\sum_{z}\left[\sum_{y} P(y) P(z / y)^{1 / 2}\right]^{2}\right)\right\}
$$

Now from above concept (15) can be written as

$$
\overline{P_{2}\left(y_{i}, y_{j}\right)} \leq 2^{-n R_{c}}
$$

\section{SIMULATION ENVIRONMENT AND RESULTS}

Here we have used a long Irregular LDPC code which answers all the questions aroused from Shanon's theorem $[1,2,11]$. LDPC code of almost any rate and block length can be designed only from the specification of target parity check matrix. This system is simulated at the transmitter side by encoding the serial stream of data by the LDPC encoder and then converting it into a QAM modulated OFDM wave and passing it into the AWGN channel or Rayleigh fading channel or Rician channel. At the receiver side, the QAM demodulator calculates the 1lr (log likelihood ratios) followed by the LDPC decoder (Hard-decision or Soft-decision) [1,2] that gives the decoded message. Here the simulation is implemented in the baseband domain using matlab coding. The LDPC code is an irregular LDPC code with parity check matrix $(32400,64800)$. Parity-check matrix of the LDPC code is stored as a sparse logical matrix. The system was simulated over the three channels with various rates as $1 / 2,1 / 3,1 / 4,2 / 3,2 / 5,3 / 4,3 / 5$, $4 / 5,5 / 6,8 / 9$ and $9 / 10$ respectively. The LDPC decoder is of Hard and Soft-decision type [1,2]. The information is binary in nature. The encoding and decoding strategies are given below.

\subsection{Encoding of LDPC codes}

The set of valid codewords are those which satisfies the parity check constraints as given below, where $\mathrm{H}$ and $\mathrm{C}$ are the parity check matrix and the codeword respectively[6,7].

$$
\left[H C^{T}\right]=[0]
$$

But, the mapping of messages to these codewords through the use of generator matrix shows how to encode the message. The $(\mathrm{j}, \mathrm{i})$ th entry of $\mathrm{G}$ (generator matrix) is ' 1 'if the $\mathrm{jth}$ message bit plays a role in determining the ith codeword. The set of all possible linear combination of the rows of $\mathrm{G}$ gives the set of codewords for the code with generator G. Hence G satisfies the following equation.

$$
G H^{T}=0
$$

The steps for encoding after designing the parity check matrix are as below:

- Put $H$ in the row-echelon form to get a new matrix as $H_{g r}$.

- Then convert $H_{g r}$ to reduced row- echelon form denoted by $H_{g r r}$.

- Then put $H_{g r r}$ into standard form :

$$
H_{g r r}=\left[\begin{array}{ll}
A & I_{N-K}
\end{array}\right] \text {, where } A \text { is }(N-K) \text { by } K
$$

binary matrix and $I_{N-K} C=\underline{m} G$ is the identity matrix of order $N-K$.

- Then the generator matrix will be $G=\left[I_{K} A^{T}\right]$

- Then the message is mapped to the codeword by the relation $C=\underline{m} G$, where $\underline{m}$ is message vector to be encoded.

\subsection{Decoding of LDPC codes}

Gallager has also provided a decoding algorithm that is typically near optimal [11]. The iterative decoding algorithms used in LDPC decoding measures the probability distribution of variables in graph based models and come under different names depending on the circumstances. But, collectively they 
are termed as message-passing (MP) algorithms [12,13]. The task of the decoder is to detect and correct the flipped bits during transmission over any channel. Every received word that does not satisfy equation (18) above will not be a codeword.

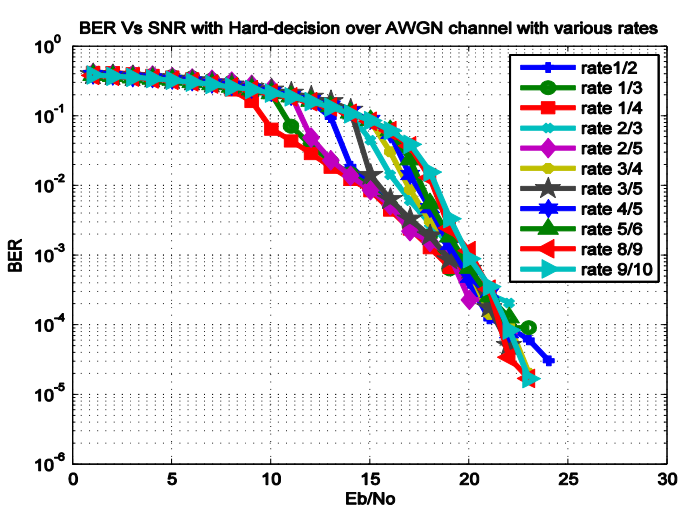

Fig1: Comparison of BER performance of LDPC coded QAM modulated OFDM wave with Hard-decision over AWGN channel with various rates

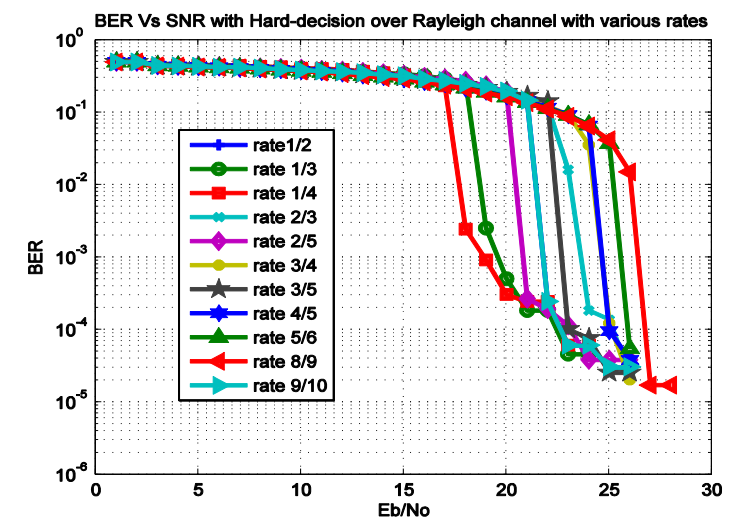

Fig2: Comparison of BER performance of LDPC coded QAM modulated OFDM wave with Hard-decision over Rayleigh fading channel with various rates

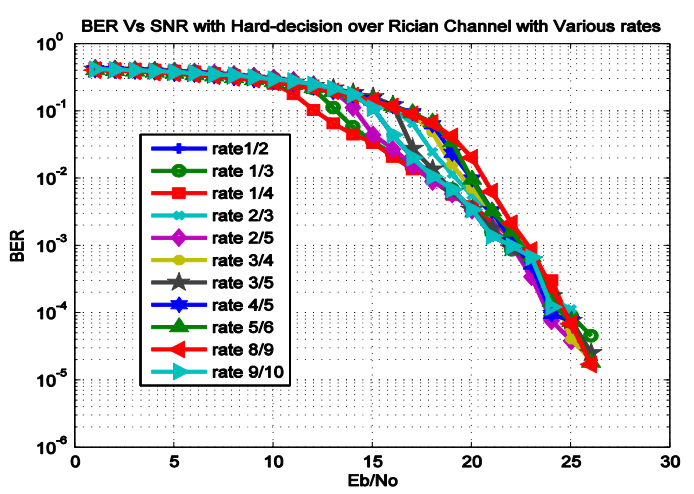

Fig3: Comparison of BER performance of LDPC coded QAM modulated OFDM wave with Hard-decision over Rician fading channel with various rates

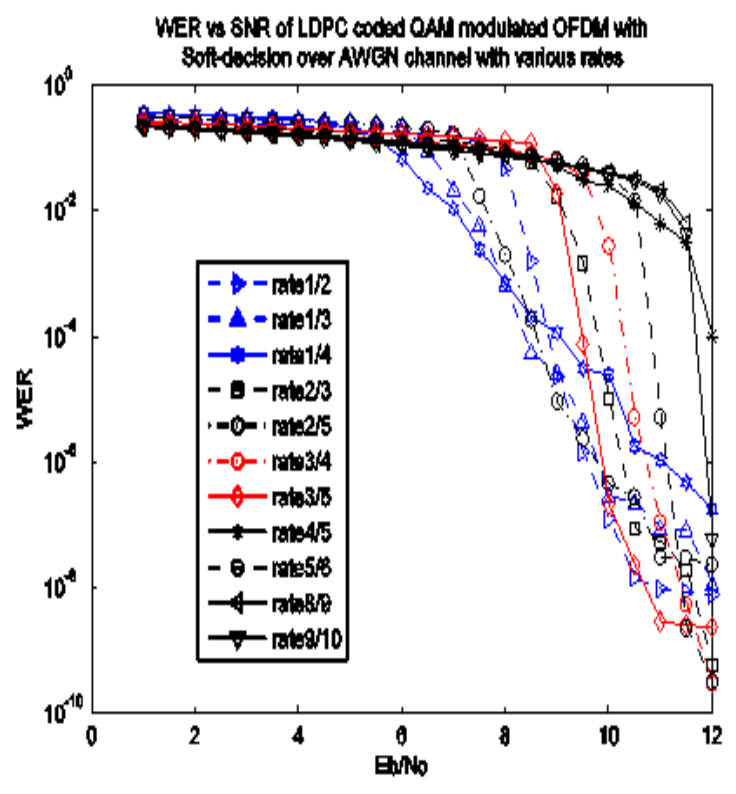

Fig4:Comparison of WER performance of LDPC coded QAM modulated OFDM wave with Soft-decision decoding over AWGN channel with various rates.

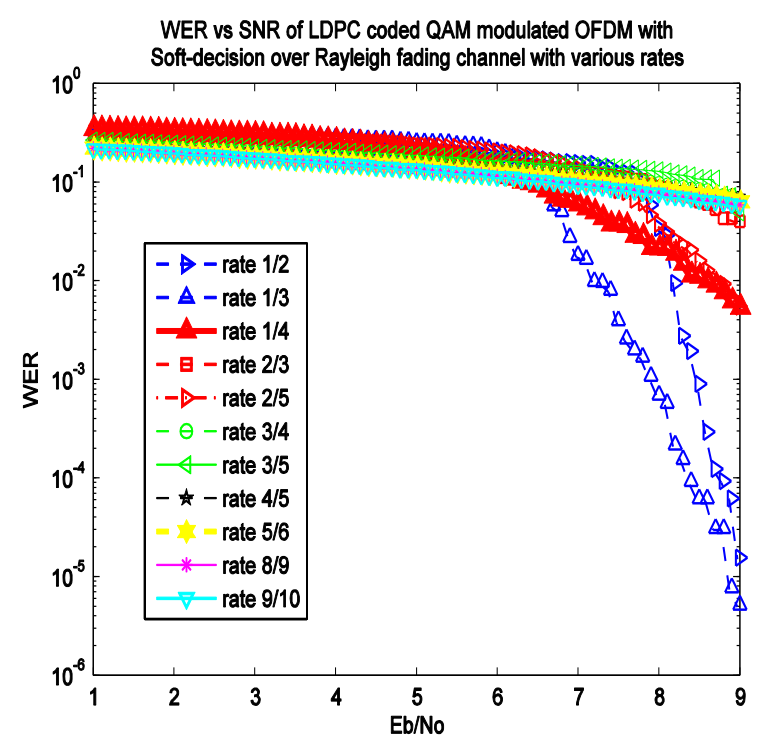

Fig5: Comparison of WER performance of LDPC coded QAM modulated OFDM wave with Soft-decision decoding over Rayleigh fading channel with various rates

In message-passing algorithm, messages pass back and forward between the bit and check nodes .The hard decision message-passing algorithm is known as bit-flipping algorithm and the passed messages are binary in nature and this algorithm is explained below. Sum-product algorithm (SPA) is the soft-decision message-passing algorithm. It is similar to the bit-flipping algorithm except that the passed messages between bit node and check node are probabilities [8]. Fig.1 compares the Bit error rate (BER) performance of the long 
irregular LDPC coded QAM modulated OFDM with various defined rates with Hard-decision decoding as mentioned above, over the AWGN channel. Figure 2 and 3 does the same over Rayleigh fading channel and Rician fading channels respectively. Fig.4 compares the Word Error rate (WER) performance of the long irregular LDPC coded QAM modulated OFDM with various defined rates with Softdecision decoding as mentioned above, over the AWGN channel. Figure 5 and 6 does the same over Rayleigh fading channel and Rician fading channels respectively.

\subsubsection{Bit-flipping Algorithm (Hard-decision Algorithm):}

For each bit $\mathrm{C}_{\mathrm{N}}$, the checks which are influenced by that bit are computed first. Then if the number of nonzero checks exceeds some thresholds, then that particular bit is decided to be incorrect and corrected by flipping it. This simple scheme is capable of correcting more than one error . Suppose that $C_{N}$ is in error along with the other bits influencing its checks. Then assuming no cycles in the tanner graph, arrange it as a tree with $C_{N}$ as a root and mark the bits which are in error [3]. The bits are said to be in tier 1 if they are connected to the checks connected to the root node. The bits that are connected to the checks from the first tier are said to be in tier 2.Many such tiers can be established likewise. Then start decoding proceeding from the leaves of the tree and by the time decoder reach at the root of the tree $\left(\mathrm{C}_{\mathrm{N}}\right)$, other erroneous bits may have been corrected. Figure 1and 2 and 3 above compares the performance of the long irregular LDPC coded QAM modulated signal with various defined rates with this Harddecision algorithm.

\subsubsection{Sum-product Algorithm (Soft-decision Algorithm):}

If we are transmitting a Codeword with $\mathrm{N}$ number of bits, then the APP is the probability that the given bit in the transmitted codeword is equal to 1 or 0 , given the channel output for that bit. Then the APP ratio or the likelihood ratio (LR) is given by

$$
l\left(c_{j}\right)=\frac{\operatorname{Pr}\left(c_{j}=0 / \text { channeloutputfor } 0\right)}{\operatorname{Pr}\left(c_{j}=1 / \text { channeloutputfor } 1\right)}
$$

Then the log-APP ratio or the log-likelihood ratio(LLR) will be given by

$$
L\left(c_{j}\right)=\log \left(\frac{\operatorname{Pr}\left(c_{j}=0 / \text { channeloutputfor } 0\right)}{\operatorname{Pr}\left(c_{j}=1 / \text { channeloutputfor } 1\right)}\right)
$$

The three key parameters in this algorithm are $L\left(r_{i j}\right)$, $L\left(q_{j i}\right)$ and $L\left(Q_{j}\right)$.

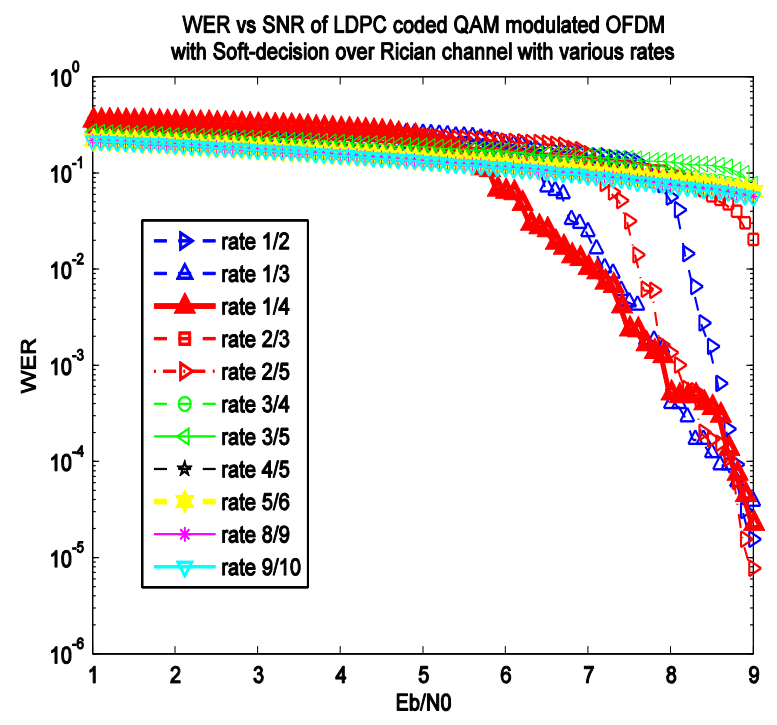

Fig6: Comparison of WER performance of LDPC coded QAM modulated OFDM wave with Soft-decision decoding over Rician fading channel with various rates.

The $L\left(q_{j i}\right)$ is initiated as $L\left(q_{j i}\right)=L\left(c_{j}\right)$ and these three parameters are updated using the following equations for each iteration $[8,12,13]$.

$$
\begin{aligned}
& L\left(r_{i j}\right)=2 a \tanh \left(\prod_{j^{\prime} \in v_{i} / j} \tanh \left(\frac{1}{2} L\left(q_{j^{\prime} i}\right)\right)\right) \\
& L\left(q_{j i}\right)=L\left(c_{j}\right)+\sum_{i^{\prime} \in c_{j} / i} L\left(r_{i^{\prime} j}\right) \\
& L\left(Q_{j}\right)=L\left(c_{j}\right)+\sum_{i \in c_{j}} L\left(r_{i j}\right)
\end{aligned}
$$

\section{CONCLUSION}

The simulation results show that with rate $1 / 4$, the performance of the code is better than all other rates in all the three cases of channels with hard-decision decoding for lower value of SNR and also the error floor region is between $10^{-4}$ to $10^{-5}$ in all the three channel cases more or less with different values of SNR between 20 to $30 \mathrm{~dB}$. While the result is obtained at slightly lower value of SNR in AWGN channel cases than the other two fading channel cases. So the performance of the code with harddecision decoding is slightly dependent on the channel type with various rates.

Similarly with rate $9 / 10$, the performance of BER plot degrades than that with all other rates in all the three channel cases and also the error floor region is between $10^{-4}$ to $10^{-5}$ in all the three channel cases more or less. So we can say that performance of the code is slightly dependent on the channel type. Hence the cut off rate here is $9 / 10$ for all the three channel types discussed. Thus through simulation results we got a cut-off rate with harddecision decoding, which will act as an information base for the practically instrumentable reliable communications.

With soft decision decoding, the scenario is little different .For the AWGN channel case, up to $7.5 \mathrm{~dB}$, performance of rate $1 / 4$ 
code is better. After $7.5 \mathrm{~dB}$, rate $1 / 4,1 / 3$ and $1 / 2$ codes are giving nearly same performance up to $8.5 \mathrm{~dB}$, keeping the error floor between $10^{-3}$ to $10^{-4}$. After $8.5 \mathrm{~dB}$ up to and $9.5 \mathrm{~dB}$ the performance of rate $1 / 3,1 / 2$ and $2 / 5$ is better than all others giving an error floor of nearly $10^{-6}$. Between $9.5-10 \mathrm{~dB}$, the rate $1 / 3$ and $3 / 5$ are giving the better performance, keeping the error floor between $10^{-7}$ to $10^{-8}$. Between $10-10.5 \mathrm{~dB}$, the rate $3 / 5$ gives better error floor than rate $1 / 3$, keeping it nearly at $10^{-9}$. At $12 \mathrm{~dB}$, the rate $2 / 5$ code is giving better error floor (nearly $10^{-10}$ ) than all other rates. But, here also the rate $9 / 10$ code is giving worst performance in both lower as well as higher SNR regions and thus giving an idea of cut-off rate in this case for the practically instrumentable reliable communications .

Similarly, with soft-decision decoding over Rayleigh fading channel, clearly it is visible that the rate $1 / 2$ and $1 / 3$ codes are giving better performance between 7-8 dB, keeping the error floor between $10^{-5}$ to $10^{-6}$. Similarly with rate $9 / 10$ code the performance is the worst. thus giving an idea of cut-off rate for the practically instrumentable reliable communications .

With soft-decision decoding over Rician fading channel, clearly it is visible that between $6-8 \mathrm{db}$ the rate $1 / 4$ code is giving better performance and between $8-9 \mathrm{~dB}$, the rate $1 / 3$ and $2 / 5$ codes are giving better performance ,keeping the error floor between $10^{-5}$ to $10^{-6}$. Similarly with rate $9 / 10$ code the performance is the worst. thus giving an idea of cut-off rate in this case for the practically instrumentable reliable communications .

\section{REFERENCES}

[1] Mishra, M.; Patra, S.K.; Turuk, A.K. ,'Performance of Power efficient LDPC coded OFDM over AWGN channel”, RAIT, March 2012, Page(s): 185 - 191.

[2] Mishra, M.; Patra, S.K.; Turuk, A.K. ,'Long Irregular LDPC Coded OFDM with Soft Decision", IJCA(09758887),vol-56,N0.4,October 2012.

[3] TODD K.MOON, “ Error Correction coding”, WilleyInterscience,2006.

[4] STEPHEN G.WILSON, "Digital Modulation and coding", Pearson Education,2003.

[5] STALlingS, “ Wireless Communications and Networks", Pearson Education,2002.

[6] T. J. Richardson and R. L. Urbanke, "Efficient encoding of low-density parity-check codes," IEEE Trans. Inform. Theory, vol. 47, no. 2, pp. 638-656,Feb. 2001.

[7] Shu Lin and Daniel J.Costello ,"Error Control Coding", Jr.second.edition, Prentice Hall, 2004.

[8] Ferrari and Raheli, "Ldpc coded Modulations", SPRINGER Publication,2009.

[9] Michele,Gianluigi and Riccardo.,'Does the Performance of LDPC Codes Depend on the Channel,"IEEE Transactions on Communications, 54(12),December 2006.

[10] Ungerboeck,G.,"ChannelcodingwithAmplitude/Phasemo dulation,'IEEECommunicationsMagazine, 25(521), 1987
[11] R.Gallager,"Low-densityparity-checkcodes,"IEEETrans. Inform.Theory, vol. 8, no. 1, pp. 21-28, Jan. 1962.

[12] Bernhard M.J. Leiner, "LDPC Codes- a brief Tutorial", April 8,2005.

[13] William E.Ryan, " An Introduction to LDPC Codes", August, 19,2003.

\section{AUTHORS PROFILE}

Ms. Madhusmita Mishra: Received B.E Degree in Electronics and Communication Engineering from Utkal University, Orissa in 1997. She creditably completed her M.E Degree in Communication Control and Networking from R.G.P.V, Bhopal in 2005. She is serving the National Institute of TechnologyRourkela, India as a Research Scholar in the Department of Electronics and Communication Engineering from 2009 onwards. Her specialization is focused on Communication System Design.

Prof. Sarat Kumar Patra: Received Bsc (Engg.) from UCE Burla in Electronics and Telecommunication Engg. discipline. After completion of his graduation he served for India's prestigious Defense Research Development Organization (DRDO) as a scientist. He completed M.Tech at NIT Rourkela (Formerly known as REC Rourkela) in Communication Engg. Specialization in 1992. He received $\mathrm{PhD}$ from University of Edinburgh, UK in 1998. He has been associated with different professional bodies such as senior member of IEEE, Life Member IETE (India), IE (India), CSI (India) and ISTE (India). $\mathrm{He}$ has published more than 70 international journal and conference papers. Currently he is working as Professor in the Department of Electronics \& Communication Engineering at NIT Rourkela. His Current research area includes mobile and wireless communication, Communication Signal processing and soft computing.

Prof. Ashok Kumar Turuk:Dr Ashok Kumar Turuk received his $\mathrm{BE}$ and $\mathrm{ME}$ in Computer Science and Engineering from National Institute of Technology, Rourkela(Formerly Regional Engineering College, Rourkela) in the year 1992 and 2000 respectively. He obtained his $\mathrm{PhD}$ from IIT, Kharagpur in the year2005.Currently he is working as Associate Professor in the Department of Computer Science \& Engineering at NIT Rourkela. His research interest includes Ad-Hoc Network, Optical Network, Sensor Network, Distributed System and Grid Computing. 